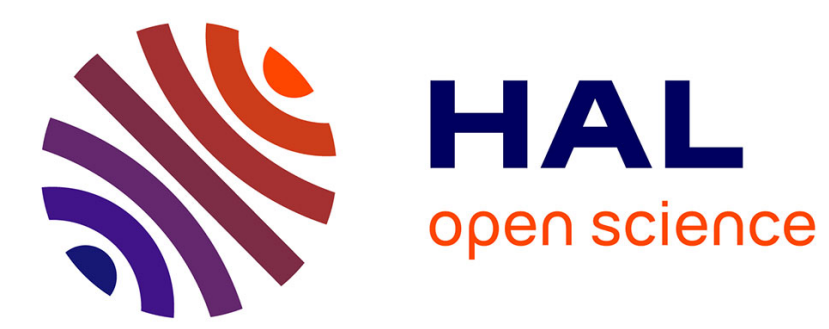

\title{
ROTATION ALIGNED NEGATIVE PARITY SIDE BANDS IN LIGHT TUNGSTEN AND OSMIUM NUCLEI
}

\author{
G.D. Dracoulis
}

\section{- To cite this version:}

G.D. Dracoulis. ROTATION ALIGNED NEGATIVE PARITY SIDE BANDS IN LIGHT TUNGSTEN AND OSMIUM NUCLEI. Journal de Physique Colloques, 1980, 41 (C10), pp.C10-66-C10-78. 10.1051/jphyscol:19801007 . jpa-00220626

\section{HAL Id: jpa-00220626 https://hal.science/jpa-00220626}

Submitted on 1 Jan 1980

HAL is a multi-disciplinary open access archive for the deposit and dissemination of scientific research documents, whether they are published or not. The documents may come from teaching and research institutions in France or abroad, or from public or private research centers.
L'archive ouverte pluridisciplinaire HAL, est destinée au dépôt et à la diffusion de documents scientifiques de niveau recherche, publiés ou non, émanant des établissements d'enseignement et de recherche français ou étrangers, des laboratoires publics ou privés. 
G.D. Dracoulis.

Department of Nuclear Physics, Research School of Physical Sciences, Australian National University, P.0. Box 4, A.C.T. Canberrra, Australia.

\begin{abstract}
Rotation aligned negative parity sidebands have been observed in the light Tungsten and Osmium isotopes. The development from octupole bands to aligned 2-quasiparticle bands is discussed. The $h_{9} / 2$ proton and $i_{13 / 2}$ neutron are the likely configurations causing the alignment. Backbending observed in the odd spin negative parity sidebands in $180^{\circ}$ os suggests that both proton and neutron configurations are involved at high spin. New results on backbending in the yrast bands of the light Osmium isotopes are also discussed.
\end{abstract}

\section{INTRODUCTION}

The study of sidebands in even-even deformed nuclei is an active field which $I$ will not attempt to review in the time available here. Rather, I will concentrate on the systematic properties of negative parity sidebands, which show the effects of rotation alignment, in Tungsten $(z=74)$ and 0smium $(\mathrm{Z}=76)$ isotopes with $\mathrm{N}=100$ to 108 . The reasons for this choice are personal aquaintance with the region, but more importantly, the large isotopic range of nuclei studied to high spin which reveals the systematic behaviour and occurrence of these bands.

I will not dwell on the details of experimental techniques, however, it is worth remembering that the population and decay pattern in (Heavy Ion, $x n$ ) reactions used to study neutron deficient nuclei preferentially populates yrast states. In deformed nuclei the yrast states are usually the ground state rotational band members up to about spin 16-20n (and about $3 \mathrm{MeV}$ excitation energy). Sidebands could be loosely defined as rotational bands with configurations different from the ground state, such as 2 (or higher) quasiparticle states or other collective states (e.g. vibrational states), which necessarily begin in the region of the pairing gap, about $1 \mathrm{MeV}$ excitation. If it were not for rotational alignment at high spin in some of these configurations, they would not compete favourably for population in the reactions. Even with rotation alignment, the negative parity bands are usually non-yrast and receive even in the most favourable cases discussed here, only a small proportion of the feeding.

Although the level schemes I will show are partial schemes, the selection of the aligned negative parity bands is not arbitrary since in the lighter isotopes $(\mathrm{N}=100$ and $\mathrm{N}=102)$ they are the strongest sidebands observed and with the exception of bands based on 2-quasiparticle isomeric states, the only sidebands identified to high spin. They are in these lighter isotopes the "yrast negative parity" states.

Octupole States and Rotation Alignment

Any discussion of negative parity states in this region is at least partly connected with octupole states, which are well known in the heavy Tungsten and Osmium nuclei. Their collective nature is established by their preferential population in $\left(d, d^{\prime}\right)$ studies and from their $\gamma$-decay which involves strong E3 transitions ${ }^{1-5)}$. Neergard and Voge ${ }^{5)}$ have successfully calculated the properties. of low-lying octupole states in a wide range of nuclei using a quasiparticle random phase approximation with an octupole-octupole residual interaction. In a deformed nucleus the $3^{-}$vibration splits into 
states with $K^{\pi}=0^{-}, 1^{-}, 2^{-}$and $3^{-}$each with rotationa1 bands, the $\mathrm{O}^{-}$band having (essentially) only odd spin members. The bands are successively coupled through large Coriolis matrix elements. Because of these interactions the bands (usually only the lowest energy band is known) are perturbed. In the Tungsten and Osmium region the lowest states are predominantly the 2-quasiproton configuration $\left\{\frac{9}{2}{ }^{-}[514] \pi, 52^{+}[402] \pi\right\}$ with some 2-quasineutron admixtures. In Tungsten the $2^{-}$configuration is usually lowest whilst in Osmium the $3^{-}$configuration is seen. Voge ${ }^{6)}$ has extended the RPA calculations to higher spin and also carried out a 2-quasiparticle calculation in a subspace including all states originating in the unique parity subshell (for protons and neutrons) and about 10 normal parity quasiparticle states close to the Fermi surface (see footnote $^{+)}$. Vogel reached specific conclusions which are relevant to the results $I$ will show. In summary these were

1] At low and intermediate spins the negative parity states are aligned octupole states. The odd and even spin members of the normal rotational bands split into separate $(\Delta J=2)$ sequences. The odd spin states are favoured in energy and have

$$
I \simeq \mathrm{R}+3
$$

while the even spin states have

$$
\mathrm{I} \approx \mathrm{R}+2
$$

where $R$ is the collective rotation $(=0,2,4 \ldots)$. That is, the octupole vibration is aligned with the rotation, and the spacing of the $\Delta J=2$ sequences is that of the ground state (" $R$ ") configuration, leading to bands with a high apparent moment of inertia. The wave functions are such that the participating quasiparticles have their intrinsic angular moments $\underset{\sim}{j}$ and ${\underset{\sim}{j}}_{2}$ almost anti-parallel.

2] At high spins ( 210 in this region) a transition to an aligned quasiparticle configuration is predicted where the particles have their spins parallel - the aligned angular momentum being dominated by the contribution of the high spin (unique parity) quasiparticle.

I will keep these predictions in mind in discussing the experimental results, when I refer to the possible 2-quasiparticle configurations, proton and neutron, that contribute to the alignment in the observed bands.

\section{Negative Parity Bands in Tungsten}

In figure 1 I have collected the partial decay schemes of high spin states in $174 \mathrm{~W}$ and $176 \mathrm{~W} 18$ ), $\left.178_{W} 19-21\right), 18 W_{W}^{22-24)}$, and $\left.182_{W} 25,26\right)$. The $2^{-}$ octupole band in $182 \mathrm{~W}$ drops in energy in going from $182 \mathrm{~W}$ to $180 \mathrm{~W}$ and as we progress to the lighter isotopes the character of the band changes in severa1 ways.

a) The odd and even spin states separate so that in the lighter isotopes the excitation energies are approximately $E_{\text {odd }}^{J} \approx E_{\text {even }}^{J}$ and the $\Delta J=1$ cascade transitions become weaker, mainly because the transition energy is reduced.

b) The decay pattern of the out-of-band El transitions changes from decays mainly from the bandhead (or states close to $i t$ ), to decays from the high spin states to the gsb, and predominantly from the odd spin states to the gsb. This out-of-band pattern, which I will comment on below, results in the population being funnelled into the gsb, before the lowest spin states in the bands can be reached. That is, the $4^{-}, 5^{-}$states seen in the lightest isotopes are not to be taken as band heads.

\footnotetext{
† Although I will not discuss them here, a number of calculations relevant to negative parity high spin states have been reported by $\mathrm{Fl}$ aum and $\mathrm{Cline}{ }^{7}$, Hjorth et. al ${ }^{8}$ ), Neergard, Vogel and Radomski ${ }^{9}$, Lin Faessler and Dreizler10), Ploszajczak and Faessler11), Krumlinde and Marshalek 12), Toki, et a1 13), Zolnowski et $a 1^{14}$ ), Hamamoto and Sagawa15), Konijn et a1 16), F.W.N. de Boer et al 17) and certainly others.
} 
c) The bands become compressed so that they have a high apparent moment of inertia. The high odd spin states $\mathrm{J}$, are at about the same excitation energy as the yrast $\mathrm{J}+1$ positive parity states in $174 \mathrm{~W}$ and $176 \mathrm{~W}$ where the negative parity states are known to high spin.

Taking these points in a little more detail,

a) The odd-even energy splitting can be seen more dramatically in a plot of $\Delta E(J \rightarrow J-1) / 2 J$ vs. $2 J^{2}$ given in figure 2. Only small oscillations are observed in $182 \mathrm{~W}$, consistent with small second order admixtures of the $\mathrm{O}^{-}$band. These develop to large oscillations for the $\mathrm{N}=100$ and $\mathrm{N}=102$ isotopes.

b) The out-of-band El transitions show several features which I can only outline here. The strength of the $J \rightarrow J-1$ El transitions can be estimated through the branching ratios in comparison with the competing in-band $\mathrm{E} 2$ transitions. The ratio
$B(E 1) / B(E 2)$ is about $10^{-6} b^{-1} 18$ ) for the odd spin states, with a trend toward larger strengths in the lighter isotopes. The single particle strengths, taking the El transition from the $9^{-}$state in ${ }^{174} \mathrm{~W}$ as an example, would be about $5 \times 10^{-5}$ Weiskopff Units, implying low $-K$ components in these states.

E1 Transitions from the even spin states are weaker. This difference has been attributed (see for example refs. $20,27,28)$ ) to the difference between $\Delta K=0$ and $\Delta K=1$ El transition strengths. At low spins $\Delta K=1$ transitions are considerably weaker than $\Delta K=0$ transitions and since the odd spin states will have both $\mathrm{K}=0$ and $\mathrm{K}=1$ admixtures (as well as $\mathrm{K}=2$ and $\mathrm{K}=3$ admixtures which will not contribute to allowed dipole decays) while the even spin states will not have $K=0$ admixtures (remembering that the $\mathrm{K}^{\pi}=0^{-}$band only had odd spin states ${ }^{\dagger}$ ), the transitions from the odd spin states will be stronger.



Figure 1 Partial level schemes showing negative parity sidebands and yrast bands in $174 W^{2} 182 W$. (see text for references) 


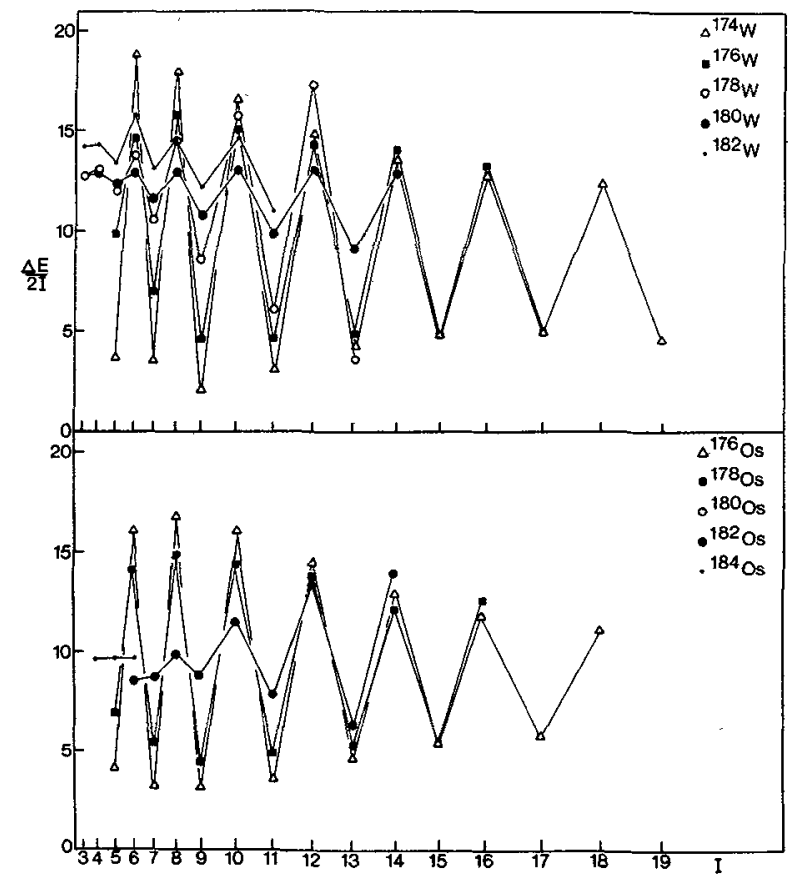

Figure $2 \quad\left(E_{J}-E_{J-1}\right) / 2 J$ vs $J^{2}$ for the negative parity bands in Tungsten and Osmium.

A further trend is evident in the branching ratios from the odd spin states. These decay by both $J \rightarrow J+1$ and $J \rightarrow J-1$ transitions to the gsb. At low spin these are comparable in intensity, despite the lower energy of the $J \rightarrow J+1$ transition. Over a sma11 range of increasing spin the situation changes to a more "normal" ratio until at high spins only $J \rightarrow J-1$ transitions are observed. The following table illustrates the trend for two of the tungsten cases. Further examples have been measured in the Osmium nuclei ${ }^{30}$ ).

This effect is probably due to a cancellation of the $\Delta K=0$ and $\Delta K=1$ components (see also ref 28)) or may indicate a change from the octupole mode which induces the El moment.

\section{c] Moments of Inertia and Rotation Aligned}

\section{Angular Momentum}

In the following discussions I will use the prescription of Bengtsson and Frauendorf ${ }^{31)}$ to deduce the aligned angular momentum $1_{x}$ and rotation- al frequency $\hbar \omega$ within a band, from the transition energies. That is

$$
\omega_{\omega}\left(I_{m}\right)=\frac{E\left(I_{m}+1\right)-E\left(I_{m}-I\right)}{I_{x}\left(I_{m}+1\right)-I_{x}\left(I_{m}-1\right)}
$$

with

$$
I_{x}\left(I_{m}\right)=\sqrt{\left(I_{m}+\frac{1}{2}\right)^{2}-K^{2}}
$$

The aligned angular momentum with respect to a reference configuration is given as

$$
i(\omega)=I_{x}(\omega)-I_{x}^{g}(\omega) \text {. }
$$

\begin{tabular}{|c|c|c|c|c|c|c|}
\hline $178_{W}$ & $J$ & $\rightarrow$ & $\mathrm{J}_{\mathrm{gsb}}$ & $I_{\gamma}$ & $\begin{array}{c}E_{\gamma} \\
(\mathrm{keV})\end{array}$ & $\frac{B(E I) J \rightarrow J+1}{B(E 1) J \rightarrow J-1}$ \\
\hline & \multirow[t]{2}{*}{5} & & 6 & 4.7 & 650 & 8 \\
\hline & & & 4 & 2.1 & 1002 & 1 \\
\hline & \multirow{2}{*}{\multicolumn{2}{|c|}{7}} & 8 & 0.7 & 514 & 1.5 \\
\hline & & & 6 & 3.1 & 962 & 1 \\
\hline \multirow[t]{6}{*}{$174 \mathrm{~W}$} & \multirow[t]{2}{*}{5} & & 6 & 3.3 & 695 & 4.9 \\
\hline & & & 4 & 2.3 & 1046 & 1 \\
\hline & \multirow{2}{*}{\multicolumn{2}{|c|}{7}} & 8 & 3.9 & 538 & 10.0 \\
\hline & & & 6 & 2.3 & 971 & 1 \\
\hline & \multirow[t]{2}{*}{9} & & 10 & $\leq 0.8$ & 361 & $<1.4$ \\
\hline & & & 8 & 8.0 & 860 & 1 \\
\hline
\end{tabular}

where $g$ refers to the reference band.

TABLE 1. Branching Ratios and $B(E 1)$ ratios.

The aligned angular momentum $I_{x}$ is shown for the ${ }^{174} \mathrm{~W}$ and ${ }^{176} \mathrm{~W}$ bands in figure 3 . The negative parity bands are seen to have an alignment higher than that of the gsb, and similar to that observed in the yrast band after backbending. By taking the gsb as the reference configuration (extrapolated where necessary through the backbending region ${ }^{\dagger}+$ ) the alignment $i$ is obtained. This is shown in figure 4 for all the Tungsten isotopes.

The $\mathrm{N}=108$ isotope ${ }^{182} \mathrm{~W}$ shows little alignment.

\footnotetext{
${ }^{\dagger}$ In the even-even nuclei the reference has been taken as the gsb. In the odd nuclei the average of the even-even neighbours has been used. There is considerable uncertainty in extrapolations through the backbending region, and this should be borne in mind when comparing relative alignments.
} 
In contrast the alignment in ${ }^{174} \mathrm{~W}$ and ${ }^{176} \mathrm{~W}$ in creases from about 1.5 and $2.5 \hbar$ in the even and odd spin states respectively at $\hbar \omega=0.12 \mathrm{MeV}$, to about $4 \pi$ in both odd and even spin states at 0.2 $\mathrm{MeV}$. The intervening isotopes show the trend of increased alignment with decreasing neutron number, correlated roughly with the energy splitting given in figure 2. We note in passing here that the alignment observed in $180 \mathrm{~W}$, which approaches $3 \mathrm{~h}$ at the highest spin observed, was interpreted recently as observation of the alignment of the octupole vibration 23 ).

Our earlier interpretation of the observed alignment was that the 2 quasiparticle states formed by coupling an $i_{13 / 2}$ neutron, and an $h_{9 / 2}$ or $p_{3 / 2}$ neutron (not rotation aligned), which are low in the neighbouring 1 quasiparticle spectra, were the dominant configurations at high spins ${ }^{18)}$. The trend through the isotopes was attributed to the expected sensitivity of the Coriolis mixing to the position of the neutron Fermi leve1. The 1 quasineutron states and 1 quasiproton states which might be involved in the negative parity configurations (since they are the lowest states in the 1 quasiparticle spectrum) are given in table 2.

$\underline{\text { TABLE } 2}$ Low Lying Proton and Neutron Configurations.

\begin{tabular}{|c|c|c|c|c|c|}
\hline & & protons & & & neutrons \\
\hline$j$ & $\pi$ & Nilsson & j & $\pi$ & Nilsson \\
\hline$h_{11}$ & & $9 / 2-[514]$ & $f_{7 / 2}$ & & $7 / 2{ }^{-}[514]$ \\
\hline$d_{5 / 2}$ & & $5 / 2+[402]$ & $\mathrm{p}_{3 / 2}$ & - & $3 / 2^{-}[521]$ \\
\hline$g_{7 / 2}$ & + & $7 / 2{ }^{+}[404]$ & $\mathrm{h}_{9 / 2}$ & - & $5 / 2^{-}[512]$ \\
\hline
\end{tabular}

$h_{9 / 2}-1 / 2-[541]^{\dagger} i_{13 / 2}+7 / 2^{+}[633]$ or $9 / 2^{+}[624]$ Because of decoupling the $5 / 2$ or $9 / 2$ states of this configuration are usually lowest in energy.

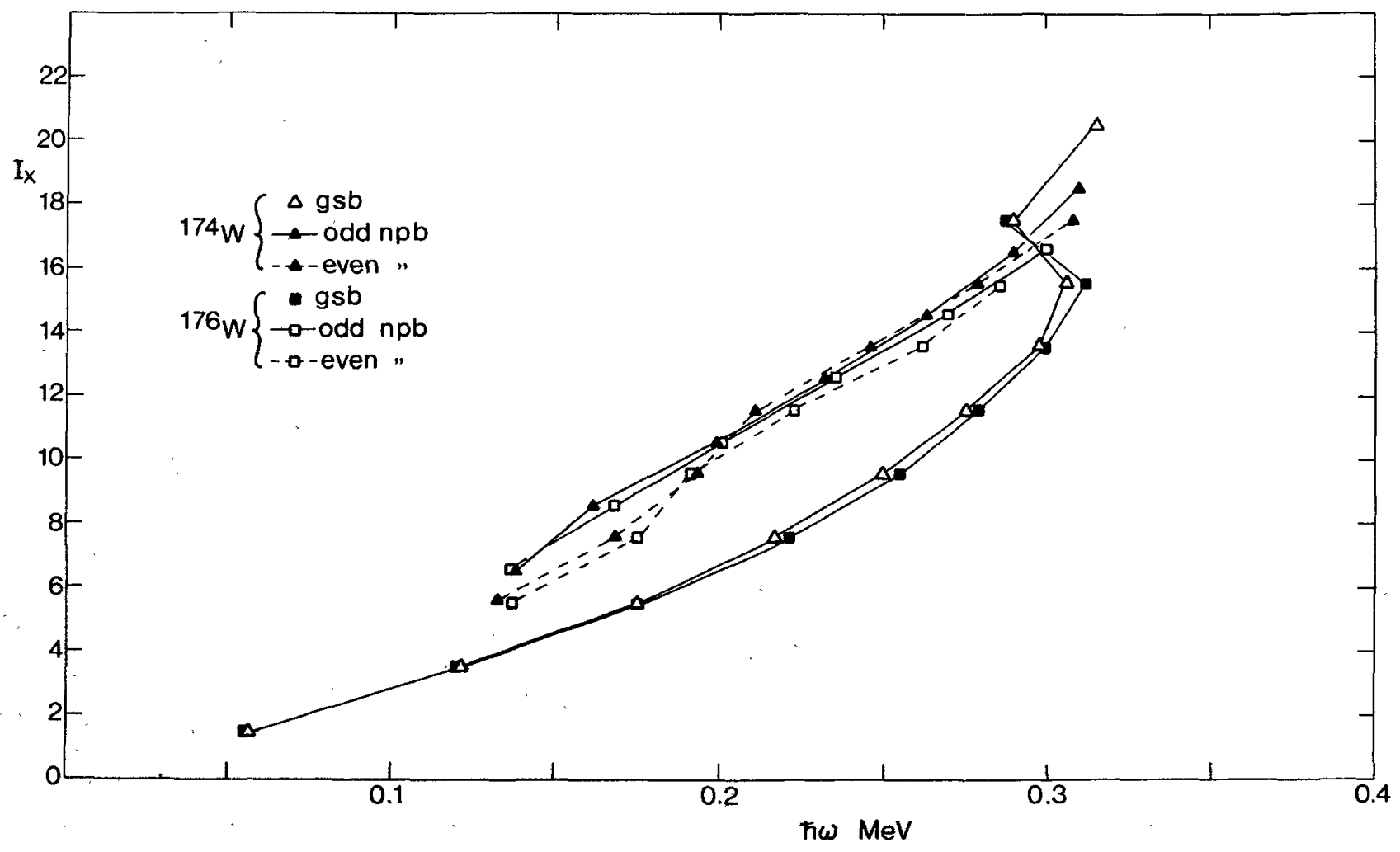

Figure 3. Aligned angular momenta $I_{x}$ (see text) vs $\hbar_{w}$ for the negative parity bands and yrast bands in ${ }^{174} \mathrm{~W}$ and ${ }^{176} \mathrm{~W}$. 
The alignments for selected bands involving these configurations can be deduced from the known spectra for $\left.\left.\left.\left.175_{W} 32\right), 177_{W} 33\right), 179_{W} 34,35\right), 181_{W} 26\right)$, and ${ }^{177} \operatorname{Re}{ }^{36)},{ }^{179} \operatorname{Re}{ }^{36)}$ and ${ }^{18 l_{\operatorname{Re}}}{ }^{37)}$. For complete-



Figure 4 (a) The aligned angular momentum i with respect to the reference configurations, for the negative parity bands in the osmium isotopes. The odd spin sequences are connected by the heavier continuous line. (b) as for (a) but for the Tungsten isotopes.

ness one should include the odd proton Ta isotopes and the heavier odd Tungsten isotopes but partly for simplicity, and partly because all the relevant data are not available, a selection has been made.

The neutron and proton alignments are shown in

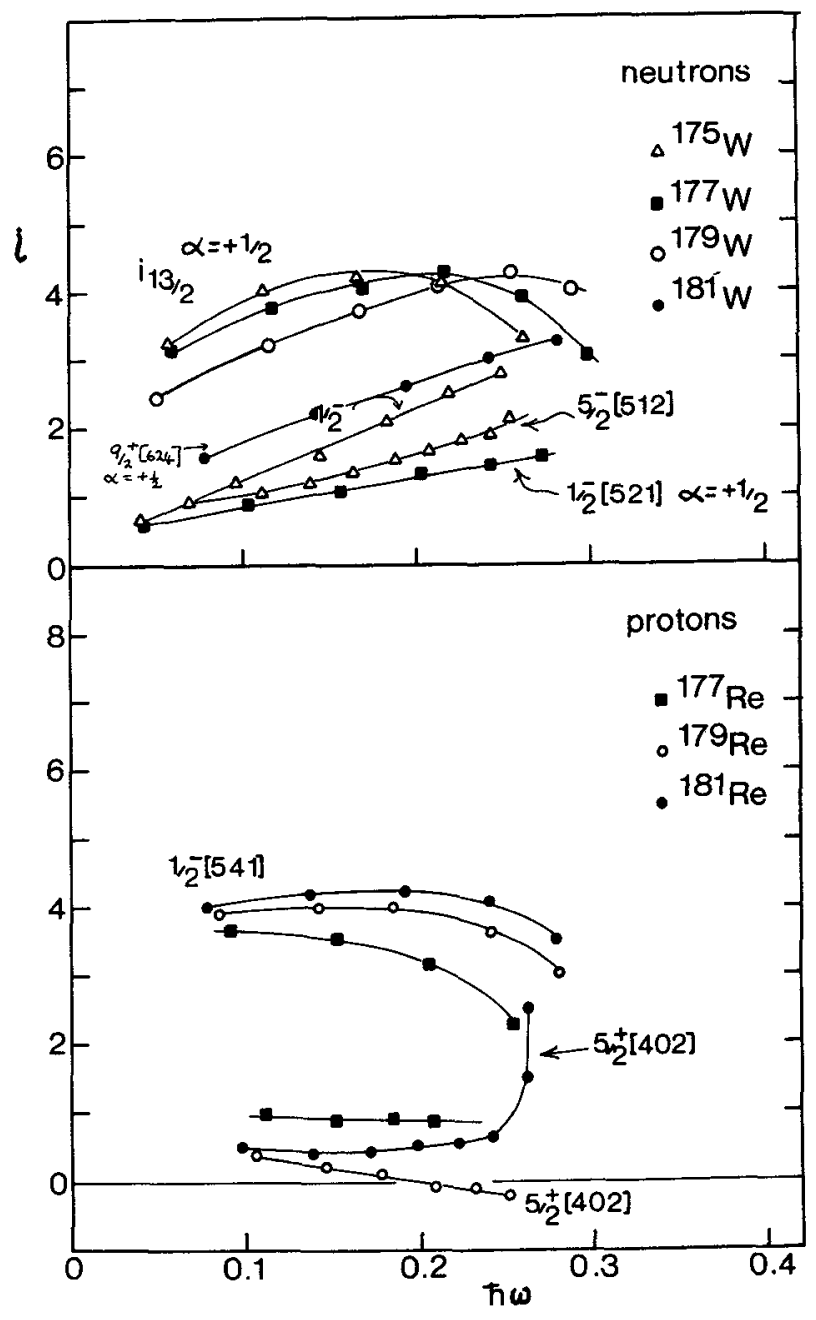

Figure 5 Relative aligned angular momenta for selected odd neutron and odd proton bands in Tungsten and Osmium. Only the $\alpha=+\frac{1}{2}$ bands are shown for configurations with a strong signature dependence.

the proton or neutron combinations could give rise to the alignments observed in the negative parity figure 5. It can be seen from these that either of bands in Tungsten since the $i_{13 / 2}$ neutron, and the $\mathrm{h}_{g / 2}$ proton are both aligned. Comparing these directly with figure 4 it is apparent that the alignpent is only reached above $\hbar \omega=0.2 \mathrm{MeV}$. Therefore, if these configurations are dominant, they are only dominant at high frequencies. At low frequencies the alignment is approximately that suggested by Vogel, 2 and $3 h$ in the even and odd spin states respectively. The transition to an aligned quasiparticle configuration is not seen in $178 \mathrm{~W}$ and the 
heaviex isotopes, at least up to the spins so far identified.

I will not attempt to make a judgement here on whether the dominant configuration at high spin is the neutron or proton one, (the neutron number dependence of the odd protons or neutron does not apparently distinguish between them) but will make further comment on that after showing the Osmium results.

Yrast bands, and Negative Parity Sidebands in light Osmium Isotopes

High spin yrast states in the light Osmium isotopes, down to neutron number 106, 1820 s, have previously been studied, particularly by the Michigan and Julich groups $38,37,39$ ). We are currently studying the isotopes from $N=100$ to $N=106$, partly with the aim of complementing the Tungsten series and will present here new results on these nuclei 30,40$)$, some of them preliminary. High spin states in Osmium were populated using $\left(16_{0}, \mathrm{xn}\right)$ reactions on enriched Er targets using ${ }^{16} 0$ beams from the ANU 14UD Pelletron accelerator. In order to facilitate the study of sidebands, as well as the usual armory of $\gamma$-ray spectrosopic techniques we have used a Compton suppressed Ge(Li) spectrometer in both singles and coincidence measurements.

Partial level schemes showing the yrast states and the rotation aligned negative parity states are shown in figure 6 , together with the results for ${ }^{184} \mathrm{Os}$ given in references 41,42$)$. Spin assignments and parities in the present study are based on angular distribution measurements and internal conversion electron measurements.

Before discussing the negative parity bands I would like to point out some features of the yrast states which have been established here up to spins of about 20 or 22 in $1760 \mathrm{~s}, 1780 \mathrm{~s}$ and ${ }^{180} \mathrm{Os}$. In ${ }^{178} \mathrm{Os}$ and $1^{180} \mathrm{Os}$, candidates for the extensions of the gsb through the backbending region have also been observed. The angular momenta $I_{x}$ shown in figure 7 for the range of isotopes from $A=176$ to 182 show a clear trend, from strong backbending in ${ }^{182} 0 \mathrm{~s}$, to a weak but well defined up-bending in 1760 s. The alignment reached at a frequency of $0.35 \mathrm{MeV}$ is about the same in each case indicating, as has been pointed out for the Tungsten isotopes ${ }^{21)}$, the common nature of the crossing band. The aligned $\left(i_{13 / 2}\right)^{2}$ neutron band is accepted as the crossing band in ${ }^{182}$ os 43 ) although earlier, the $h_{\frac{9}{2}}$ proton configuration was suggested to be responsible $\mathrm{e}^{37)}$. Further evidence that the bandcrossing is consistent with the behaviour of the $i_{1 \frac{3}{2}}$ neutron is given in the relative aligned angular momenta of figure 8 . As was seen from figure 7 , the bandcrossing occurs at progressively higher frequencies with decreasing neutron number, but the important point to note is that the alignments of the $i_{13 / 2} 1$ quasiparticle bands in $1810 \mathrm{~s}$, and from our recent study ${ }^{40)}$, in ${ }^{177} \mathrm{Os}$, are consistent with the observed alignments in the even nuclei. For simplicity, the $i_{13 / 2}$ band in ${ }^{179} \mathrm{Os}$, which we have also identified is not included on the plot. More detail on the odd neutron behaviour in the Osmium nuclei is given in figure 9. The $\frac{1}{2}^{-}[521]$ bands in ${ }^{181} 0 \mathrm{~s}$ and ${ }^{177} \mathrm{Os}$ show anomalies consistent with the magnitudes of the alignment gains observed in the yrast bands of the even neighbours, although at slightly lower frequencies. The $i_{13 / 2}$ bands, labelled $" 9 / 2+[624] "$, show anomalies only at higher frequencies, Lieder ${ }^{44}$ ) has suggested that the anomaly in the $\frac{9}{2^{+}}[624]$ band in ${ }^{181} 0$ s is due to a crossing with the $i_{13 / 2}$ three quasineutron band, but involving the $7 / 2+[633]$ neutron as well as the $\alpha= \pm \frac{1}{2}$ components of the $9 / 2^{+}[624]$ neutron. The situation in the osmium nuclei differs apparently from other cases where the blocking of the appropriate $i_{13 / 2}$ neutron delays band- 

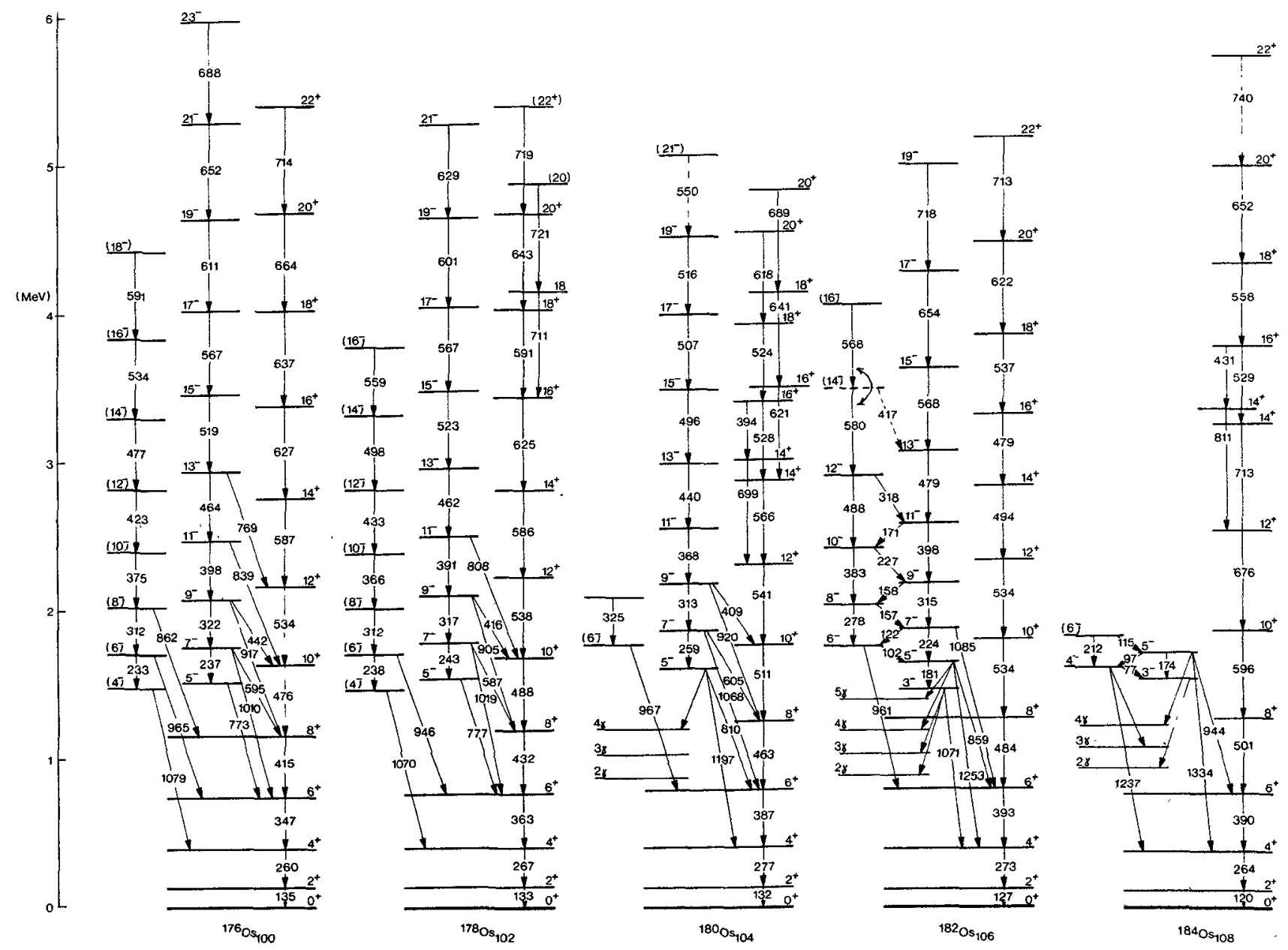

Figure 6 Partial level schemes for ${ }^{176} 0 \mathrm{~s}-{ }^{184} 0 \mathrm{~s}$. (see text for references)

crossing to considerably higher frequencies.

The tempering of the magnitude of the bandcrossing in the light os isotopes may be a demonstration of the effect of increased interaction matrix elements between the g.s. and the crossing band configurations - small matrix elements leads to strong backbending - which are predicted to have a strong mass dependence $31,45-47)$. However, calculations which correctly treat the rapid change in hexadecapole deformation which occurs in the region are required to properly examine this point. The difference in behaviour between the $\alpha= \pm \frac{1}{2}$ signatures of the $9 / 2+[624]$ band observed in ${ }^{177} \mathrm{Os}$ and ${ }^{179} \mathrm{Os}$ may be a further consequence of the same effect ${ }^{46)}$.

To return to the negative parity bands, we can follow a development similar to that observed in the Tungsten isotopes. The $3^{-}$octupole band identified in ${ }^{184} \mathrm{Os}$, unfortunately not to high spin, decays mainly to the $\gamma$-band. In 1820 s a small energy favouring of the odd spin negative parity states is observed with out-of-band decays to both the gsb and to the $\gamma$-band. Lieder et al ${ }^{51)}$ have also presented a level scheme for ${ }^{182} 0$ s in a contribution to this conference, in which they have identified a large number of bands including the negative parity band being discussed here. Our results ${ }^{30}$ ) agree with 


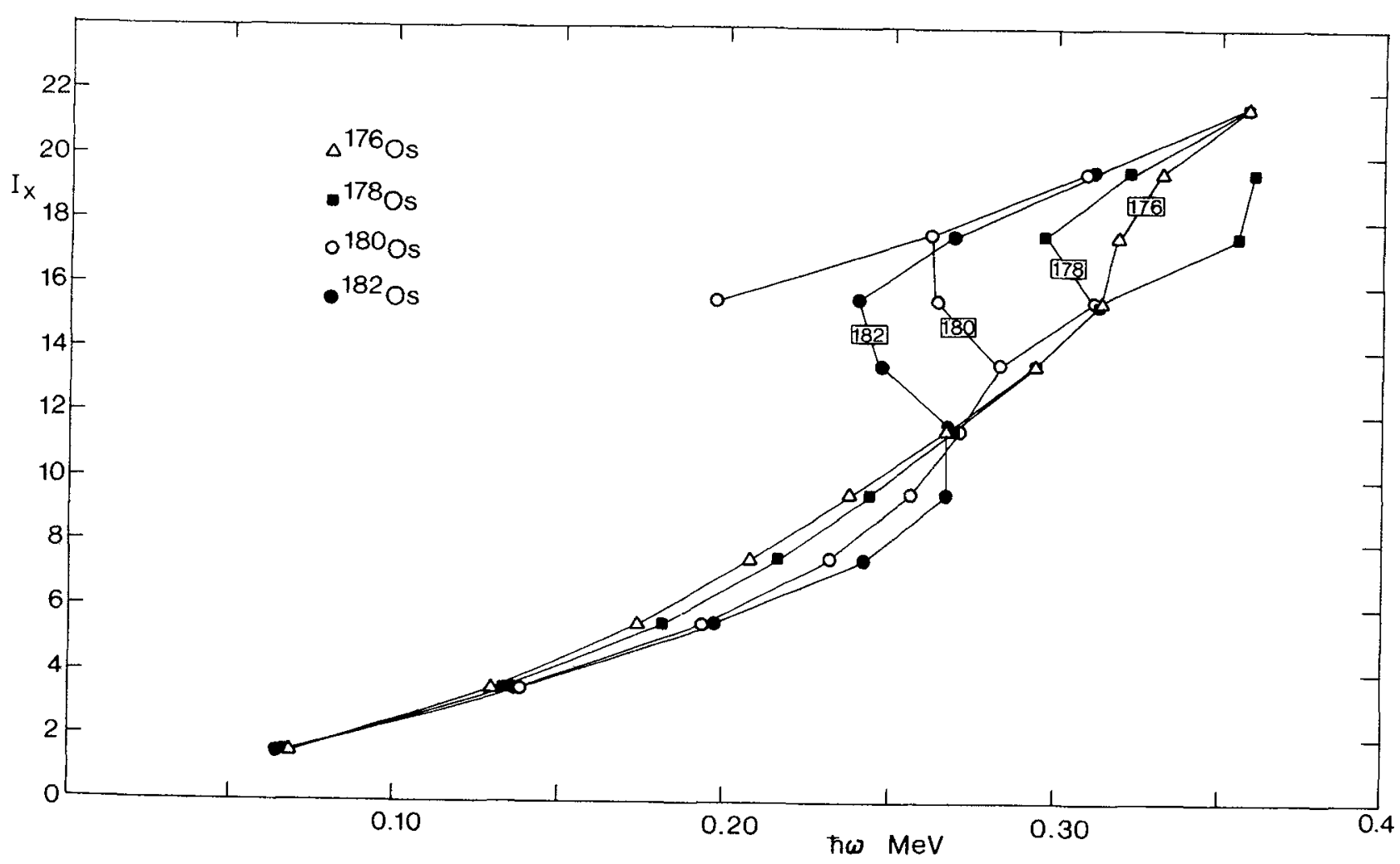

Figure 7 The aligned angular momentum $I_{x}$ vs hw for the yrast (and yrare) sequences in ${ }^{176} 0 \mathrm{~s}-{ }^{182} \mathrm{Os}$.

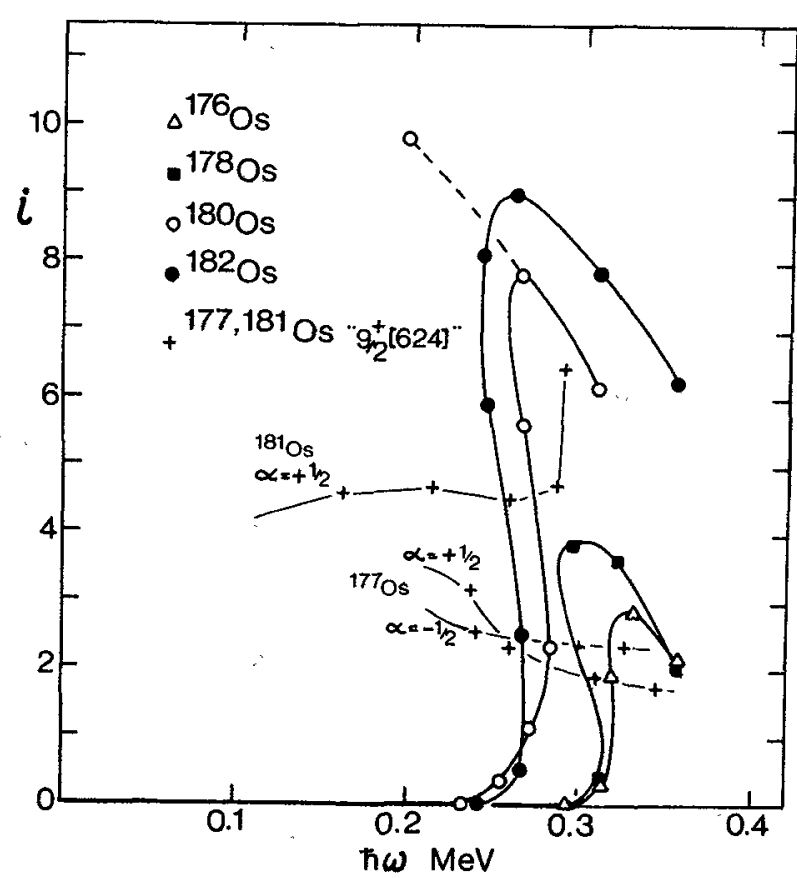

Figure 8 The relative aligned angular momenta $i$ vs $\hbar \omega$ for the yrast (and yrare) sequences in ${ }^{176} 0 \mathrm{~s}-{ }^{182} 0 \mathrm{~s}$.

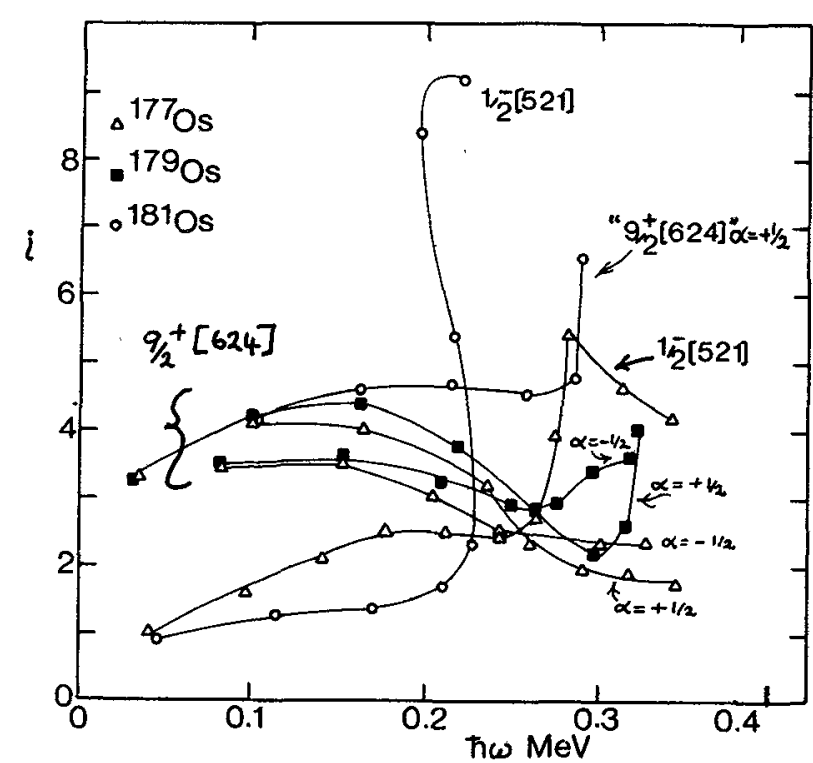

Figure 9 The relative aligned angular momenta i vs $\hbar \omega$ for selected odd neutron bands in Osmium. 
theirs on all counts except for the placement of the odd spin sequence in the present negative parity band. In both schemes, an anomaly is seen in the even spin sequence at a low frequency. In ${ }^{180} 0$ s only an odd spin sequence has been identified, (although a candidate for the $6^{-}$state is observed) with the main branches to the gsb, but still with significant branching to the $\gamma$-band. An anomaly in the band spacing is seen at about spin 17. Similar, well developed, odd spin sequences, and separate, more weakly populated even spin sequences are observed in both of the lighter isotopes 1760 s and 1780 s.

These bands are, at least superficially, remarkably similar to those in the Tungsten isotopes. The El strengths and branching ratios are similar, the odd-even energy spacing given in figure 2 is similar, but there are differences in the alignments.

The alignments are shown in figure $4 .{ }^{176}$ Os and ${ }^{178}$ os show higher alignment in the odd spin states than the even spin states at low frequencies, rising to about $4 \hbar$ at $\hbar \omega=0.18 \mathrm{MeV}$, similar to the behaviour in the $\mathrm{N}=100$ and $\mathrm{N}=102$ isotopes of Tungsten. ${ }^{180} \mathrm{OS}$ and ${ }^{182} \mathrm{Os}$ however show even larger alignments, in marked contrast to their Tungsten isotones, and in ${ }^{180} 0$ s a large alignment gain is observed at a frequency of $0.25 \mathrm{MeV}$.

The same difference is partly seen in comparing the odd-neutron isotopes of Osmium and Tungsten but it is not possible here to conclusively identify the quasiparticle configurations responsible for the alignment observed at $\hbar \omega \geq 0.2 \mathrm{MeV}$. It may be that the $h_{\frac{g}{2}}$ protons still play a significant role since there are two main differences evident in the comparison between Osmium and Tungsten. Firstly, the heavy Tungsten isotopes, $N \geqslant 106$, do not show large alignment. If the protons were responsible, this might be attributable to the difference in the
1 quasiproton spectrum, and in particular its neutron number dependence, between the Re and $\mathrm{Ta}$ isotopes. An examination of those spectra $49-50$ ) shows that the $\frac{1 / 2}{2}[541] h_{9 / 2}$ proton has, as expected, a similar alignment in the $\operatorname{Re}$ and $\mathrm{Ta}$ isotopes, but is at considerably higher excitation energy in the heavy $\mathrm{Ta}$ isotopes than in the isotonic Re nuclei. For example, in $\mathrm{Re}^{181}(\mathrm{~N}=106)$ the $\frac{5}{2^{-}}$state of the $\frac{7}{2}-[541]$ configuration is at $357 \mathrm{keV}$ while in ${ }^{179} \mathrm{Ta}$ it is at $628 \mathrm{keV}$. The aligned 2 quasiproton configuration in Tungsten, therefore, may be at a higher excitation energy, in the heavier Tungstens, than in the isotonic Osmium nuclei - al though the light nuclei may be similar. Secondly, the negative parity band in 180 os shows backbending at a low frequency. If the $i_{13 / 2}$ neutron configuration were responsible for the alignment at $0.2 \mathrm{MeV}$, then it could not be responsible for the gain in alignment at $0.25 \mathrm{MeV}$ because of blocking. The effect of blocking of the $i_{13 / 2}$ neutron is apparent in the behaviour of the $\frac{9}{2}{ }^{+}[624] 1$ quasineutron band in ${ }^{179} \mathrm{Os}$ (shown in figure 9) which does not show an anomaly until a considerably higher frequency than the $\frac{1}{2}-[521]$ band. However, the same argument could be applied to the $h_{\frac{9}{2}}$ proton configuration since in ${ }^{181} \operatorname{Re}$ the $1 / 2-[541]$ band also does not show backbending while the $5 / 2^{+}[402]$ band does, and at the same frequency, $0.25 \mathrm{MeV}$, as the negative parity band in $1800 \mathrm{~s}$. The solution to this dilemma is to postulate a change in configuration from a 2 quasiproton' configuration (involving the $h_{\frac{9}{2}}$ proton) to one involving an extra pair of $\dot{i}_{1 / 2}$ neutrons resulting in the alignment gain at $\hbar \omega=0.25 \mathrm{MeV}$. A similar explanation has been given by Ploszajcak and Faessler 11) for the backbending observed in the negative parity band of ${ }^{156} \mathrm{Er}$.

The anomaly in the negative parity even spin sequence in ${ }^{182}$ Os occurs at a frequency of about 
$0.29 \mathrm{MeV}$, higher than the anomaly in the gsb, and at about the same frequency as the $i_{13 / 2}$ band in ${ }^{181} 0 \mathrm{~s}$. Lieder et al ${ }^{51}$ ) use this as eyidence that the $i_{13 / 2}$ neutrons are responsible for the gain in alignment. However in $180^{\circ}$ s the situation is different since the anomaly in the odd spin sequence occurs at a lower frequency than that in the gsb, or in the $i_{13 / 2}$ neutron band in ${ }^{179} \mathrm{Os}$.

Certainly, the situation may be complex, and specific calculations for proton and neutron configurations for this range of Tungsten and Osmium isotopes which properly treat the changing quadrupole and hexadecapole deformations are imperative if a clearer understanding is to be reached.

To. return briefly to the introduction of this talk, and the predictions of Vogel; it seems possible to trace the development of the octupole band into separate odd and even spin sequences over a wide range of isotopes. The alignment at low frequencies is consistent with contributions of about $2 \hbar$ and $3 \hbar$ to the even and odd spin states respectively, due presumably to the alignment of the octupole spin. A transition to a band with a higher alignment is seen in most cases. This gain in alignment is consistent with that due to the $i_{1 / 2}$ neutron or the $h_{\frac{9}{2}}$ proton which could contribute to the dominant 2-quasiparticle configurations. Although it is not possible at present to definitively characterise the negative parity bands at high spin, the systematic trends, and the backbending observed in the negative parity band in ${ }^{180} 0 \mathrm{~s}$, suggest that the $i_{13 / 2}$ neutrons and $h_{9 / 2}$ protons both play a role.

I trust that I have convinced you that rotation aligned negative parity bands are a systematically occurring feature of sidebands in this region of the periodic table.
I would like to express my appreciation to my resilient colleagues Dr P.M. Walker, and Dr C. Fahl ander. 


\section{REFERENCES}

1. C. Günther, P. KIeinheinz, R.F. Casten and B. Elbek. Nuc1.Phys. A172 (1971) 273 and references therein.

2. B. Elbek, T. Grotda1, K. Nyb $\phi, P .0 . T j \phi m$ and E. Veje. Proc. Int. Conference on Nuclear Structure Tokyo, 1968, pl 80 Phys. Soc. Japan.

3. P. Herzog, M.J. Canty and K.D. Killig. Nucl. Phys. Al87 (1972) 49.

4. J. Konijn, B.J. Meijer and G. Van Middelkoop. Phys. Lett. 35B (1971) 567.

5. K. Neergard and P. Vogel. Nucl. Phys. Al45 (1970) 33.

6. P. Vogel. Phys, Lett 6OB (1976) 431.

7. C. Flaum and D. Cline. Phys. Rev. C 14 (1976) 1224.

8. S.A. Hjorth, A. Johnson, Th. Lindblad. L. Funke, P. Kemnitz and G. Winter. Nuc1. Phys. A262 (1976) 328.

9. K. Neergard, P. Vogel and M. Radomski. Nucl. Phys. A238 (1975) 199.

10. L. Lin, A. Faessler, and R.M. Dreizler. Nuc1. Phys. A318 (1979) 287.

11. M. Ploszajczak and A. Faessler. 2. Physik A283 (1977) 349.

1.2. K. Krumlinde and E.R. Marshalek. Nucl.Phys. A275 (1977) 395 .

13. H. Toki, K. Neergard, P. Vogel and A. Faessler. Nuc1. Phys. A279 (1977) 1.

14. D.R. Zolnowski, T. Kishimoto, Y, Gono and T.T. Sugihara. Phys. Lett. 55B (1975) 453.

15. I. Hamamoto and H. Sagawa. Phys. Lett $\underline{85 B}$ (1979) 177 and Nuc1. Phys. A327 (1979) 99.

16. J. Konijn, F.W.N. de Boer, P. Koldewijn, R. Beetz and L.K. Peker. Z. Physik A284 (1978) 233.
17. F.W.N. de Boer, L.K. Peker, P. Koldewijn and J. Konijn. Z, Physik A284 (1978) 267.

18. G.D. Dracoulis, P.M. Walker and A. Johnston. J. Phys. $\underline{G}$ : Nuc1. Phys. $\underline{4}$ (1978) 713.

19. M.J. Canty, N.E. Davidson, D.A. Dohan and P. Yuen. Nuc1. Phys. A265 (1976) 1.

20. C.L. Dors, F.M. Bernthal, T.L. Khoo, C.H. King, J. Borggreen and G. Sletten. Nucl. Phys. A314 (1979) 61 .

21. G.D. Dracoulis and P.M. Walker. Phys. Rev. C 20 (1979) 1619 .

22. S.R. Faber, F.M. Bernthal, T.L. Khoo and R.A. Warner. Cyclotron Laboratory. Michigan State University, Annual Report 1977/1978 p. 49 .

23. R.K. Shel ine, L.G. Mann, G.L. Struble, R.G. Lanier and J.B. Carlson. Phys. Rev. Lett. 41 (1978) 374 .

24. L.G. Mann, J.B. Carlson, R.G. Lanier, G.L. Struble, W.M. Buckley, D.W. Heikkinen, I.D. Proctor and R.K. Sheline. Phys. Rev. C 19 (1979) 1191 .

25. B.D. Jeltema, F.M. Bernthal, T.L. Khoo and C.L. Dors. Nuc1. Phys. A280 (1977) 21.

26. F.M. Bernthal, C.L. Dors, B.D. Jeltema, T. L. Khoo and R.A. Warner. Phys, Lett. 64B (1976) 147.

27. T.L. Khoo, J.C. Waddington, Z. Preibis $z$ and M.W. Johns. Nuc1. Phys. A202 (1973) 289.

28. F.W.N. de Boer, P. Koldewijn, R. Beetz, J.L. Maarleveld, J. Konijn, R. Janssens and J. Vervier. Nuc1. Phys. A290 (1977) 173.

29. V.M. Mikhailov. Bull. Acad. Sci USSR (Physical Series) Translation 32 (1968) 784.

30. G.D. Dracoulis, C. Fahlander and M.P. Fewell, to be published. 
31. R. Bengtsson and S. Frauendorf. Nuc1. Phys. A327 (1979) 139.

32. P.M. Walker, G.D. Dracoulis, A. Johnston, J.R. Leigh, M.G. Slocombe and I.F. Wright. J. Phys. G : Nucl. Phys. 4 (1978) 1655.

33. C.L. Dors, Ph.D. Dissertation. Michigan State University 1976.

34. F.M. Bernthal and R.A. Warner. Phys. Rev. C 11 (1975) 188 .

35. F.M. Bernthal, B.B. Back, 0. Bakander, J. Borggreen, J. Pedersen, G. Sletten. H. Beuscher, D. Haenni and R. Lieder. Phys. Lett. 74B (1978) 211.

36. J.R. Leigh, J.0. Newton, L.A. E11is, M.C. Evans and M.J. Emmott. Nucl. Phys. A183 (1972) 177 .

37. A. Neskakis, R.M. Lieder, M. Müller-Veggian, H. Beuscher and W.F. Davidson. Nuc1. Phys. A261 (1976) 189 .

38. R.A. Warner, F.M. Bernthal, J.S. Boyno, T.L. Khoo and G. Sletten, Phys. Rev. Lett. 31 (1973) 835 .

39. W.F. Davidson, R.M. Lieder, H. Beuscher,

A. Neskakis, Z. Seres and C. Mayer-Böricke.

Z. Phys. 264 (1973) 235.

40. G.D. Dracoulis and C. Fahlander. To be published

41. R. Hochel, P.J. Daly and K.J. Hofstetter. Nuc1. Phys. A211 (1973) 165.

42. S.W. Yates, J.C. Cunnane, R. Hoche1 and P.J. Daly. Nucl. Phys. A222 (1974) 301.

43. A. Faessler, M. Ploszajczak and K.R. Sandhya-Devi. Nucl. Phys. A301 (1978) 382 .

44. R.M. Lieder. Invited Paper. International Conference on Band Structure and Nuclear Dynamics, New Orleans, 1980.
45. R. Bengtsson, I. Hamamoto and B. Mottelson, Phys. Lett. 73B (1978) 259.

46. J. Almberger, I. Hamamoto and G. Leander. Phys. Lett. 8OB (1979) 153.

47. F. Grummer, K.W. Schmid and A. Faessler. Nucl. Phys. A326 (1979) 1 .

48. C. Foin, Th. Lindblad, B. Skanberg and H. Ryde. Nucl. Phys. A195 (1972) 465.

49. B. Skanberg, S.A. Hjorth and H. Ryde. Nucl. Phys. A154 (1970) 641.

50. D. Barneoud, C. Foin, A. Baudry, A. Gizon and J. Valentin. Nucl. Phys. Al54 (1970 654 .

51. R.M. Lieder, G. Sletten, O. Bakander, S. Bjornholm, J. Borggreen, J. Pedersen. Contribution to this conference. 